\title{
Lumen
}

Selected Proceedings from the Canadian Society for Eighteenth-Century Studies

\section{Exils et frontières dans l'oeuvre de Prévost}

\section{Jean Sgard}

Volume 16, 1997

Freedom and Boundaries

Émancipation et frontières

URI : https://id.erudit.org/iderudit/1012437ar

DOI : https://doi.org/10.7202/1012437ar

Aller au sommaire du numéro

Éditeur(s)

Canadian Society for Eighteenth-Century Studies / Société canadienne d'étude du dix-huitième siècle

\section{ISSN}

1209-3696 (imprimé)

1927-8284 (numérique)

Découvrir la revue

Citer cet article

Sgard, J. (1997). Exils et frontières dans l'oeuvre de Prévost. Lumen, 16, 25-34. https://doi.org/10.7202/1012437ar d'utilisation que vous pouvez consulter en ligne.

https://apropos.erudit.org/fr/usagers/politique-dutilisation/ 


\section{Exils et frontières dans l'oeuvre de Prévost}

Comparés aux romans de ses contemporains, Marivaux, Crébillon, Duclos, ou de ses successeurs, Rétif, Baculard ou Mercier, les romans de Prévost sont ouverts sur le grand large. Dans un siècle où l'univers romanesque, en France au moins, tend à se confiner dans Paris pour y permettre une observation fine du fait social, Prévost semble bien être le seul à ouvrir ses fenêtres sur le vaste monde. On avait connu avant lui, et particulièrement à la fin du dix-septième siècle, une ouverture du roman sur les différentes nations, sur tous les genres de l'aventure picaresque, sur la diversité des classes sociales et des modes de vie, voire sur les autres mondes et les sociétés utopiques; à partir des années 1730, Lesage est un survivant, et Prévost semble bien être le seul à se souvenir de Veiras ou de Courtilz de Sandras. Lui seul développe l'aventure à travers les grands périples européens, méditerranéens ou atlantiques, et cela dans toute son oeuvre, des Mémoires d'un homme de qualité au Monde moral. On sait qu'à partir de 1746 il se lance en même temps dans l'immense entreprise de l'Histoire générale des voyages. Le seul goût des voyages ne peut pourtant expliquer cette tendance permanente de la création romanesque chez Prévost; on observera au contraire qu'entre les voyages réels et les voyages romanesques il établit une sorte de contraste. Le voyage romanesque illustre une aventure intérieure, une quête du bonheur et peut-être un malheur essentiel qui n'ont rien à voir avec l'intérêt solidement financier du voyage réel. Il convient donc de chercher le pourquoi de ces exils, de ces voyages, de ces vies partagées entre deux pays, de ces retours malheureux vers des origines incertaines.

\section{Exils}

L'exil est dans l'oeuvre de Prévost plus qu'un thème, c'est un emblème. On sait qu'au début de 1731, au moment de publier en Hollande les tomes V à VII des Mémoires d'un homme de qualité, il a pris le nom de «Prévost d'Exiles», beau nom de plume, mais dont le sens est en luimême énigmatique. ${ }^{1}$ Ce nom évoque $d^{\prime}$ abord un lieu, la petite forteresse 
d'Exilles, aux confins du Briançonnais, qui eut le triste avantage, comme la forteresse de Pignerol, d'être pendant des siècles tantôt française, tantôt italienne: un nom prédestiné. Prévost y fut peut-être officier, à moins qu'il n'ait déserté avant. Quand il choisit son nom de plume, il pense surtout à son exil présent, en Hollande; c'est ce que dit assez noblement, et non sans lyrisme, le «Projet d'une nouvelle traduction de l'Histoire de M. de Thou» en 1731: «Expatrié, séparé de mes amis et de mes proches, abandonné du plus grand nombre, qui croira que mon coeur ignore ce que c'est que la haine...» (VII: 292). ${ }^{2}$ Mais il n'est pas sûr qu'il ne fasse pas valoir ici le privilège, propre aux écrivains réfugiés en Hollande, de pouvoir écrire librement et de répliquer vertement à leurs adversaires: l'exil, hors d'un pays enchaîné, c'est aussi la liberté. Entre un exil obligé et un exil volontaire, la différence est grande; or, chez Prévost, l'un cache souvent l'autre. Tous ses exils, en Hollande avant 1720, en Angleterre en 1728, en Hollande en 1730, en Allemagne en 1740, lui furent imposés par les circonstances; dans son oeuvre romanesque, la fuite prendra le sens d'un engagement existentiel. Ce ne sont pas moins des exils. Si l'on excepte les Voyages de Robert Lade, qui sont plus proches du recueil de récits de voyage que du roman, tous ses narrateurs se lancent comme à regret sur les grandes routes. Ce ne sont pas des voyageurs de vocation, des conquérants, mais plus souvent des fugitifs ou des transfuges. Les fugitifs obéissent aux motivations les plus diverses: tel a dû changer de pays pour rester fidèle à son souverain, au moment d'un partage entre nations; tel autre est chassé de sa famille à la suite d'un remariage de son père; mais il arrive aussi, comme dans les romans traditionnels, qu'on s'enfuie pour épouser sa maîtresse, ou pour échapper aux conséquences d'un duel malheureux. ${ }^{3}$ Les Mémoires $d^{\prime} u n$ homme de qualité sont pleins de ces errances accidentelles; la vie de $l^{\prime}$ Homme de qualité en est tissée. Un résumé de la carrière de son ami Rosambert est plus éloquent encore:

Le comte de Rosambert continua de me raconter la suite de sa vie, c'est-à-dire le fameux duel où il eut encore le malheur de tuer un de ses ennemis; sa fuite dans les pays étrangers; ses diverses courses; son arrivée en Allemagne, où il se mit au service de l'Empereur. (I: 47)

En quelques lignes, voilà évoquée une destinée prévostienne: on ne cesse pas de fuir. L'exemple de Cleveland est plus frappant encore. Poursuivi par un père sanguinaire, Cromwell, il disparaît dans une caverne du Devonshire, en resurgit pour passer en France; mais Cromwell menaçant les opposants expatriés, il faut fuir en Amérique; commence alors une longue errance, dominée par la peur du tyran, et qui conduit le héros au fin fond des Appalaches. Réfugié en France, après avoir appris par 
hasard la mort de Cromwell, il ne connaîtra pas pour autant le repos: au moment où commence la persécution des protestants, il devient suspect, ses enfants lui sont enlevés; son sort s'apparente alors à celui de Clarendon, victime des caprices de Charles II, «forcé de chercher un asile hors de [sa] patrie» et opposant irréductible à la monarchie restaurée (C 520). ${ }^{4}$ La même hantise de fuite réapparaît dans bien des oeuvres de Prévost, sous des formes différentes: dans l'Histoire de Marguerite d'Anjou, la reine ne cesse pas de fuir ses ennemis, d'échapper à des pièges pour tomber dans d'autres; dans le Monde moral, on verra d'autres proscrits: le prince Ragotzky, son ministre Brenner, et les révoltés hongrois tenter par tous les moyens d'échapper aux Autrichiens et de protéger Mlle Tekeli, héritière du trône de Hongrie.

Dans le monde convulsif de la fin du dix-septième siècle se multiplient les crises politiques et religieuses. On trouvera donc dans les romans de Prévost des exilés protestants à Londres ${ }^{5}$ ou des catholiques irlandais à Saint-Germain-en-Laye, des jacobites réfugiés en Amérique, ou des protestants clandestins à Paris. Les partages politiques s'accompagnent de difficiles problèmes économiques, car il n'est pas rare qu'un réfugié prétende encore aux biens qu'il a laissés dans son pays d'origine; ils font naître aussi des problèmes d'alliance, de mariage impossible, de rapatriements compliqués. ${ }^{6}$ Prévost, en décrivant les crises de la fin du Grand Siècle, a choisi délibérément la complexité; mais la situation se complique encore avec ceux qui passent d'un camp à l'autre.

Les transfuges offrent une richesse incomparable de situations. On peut être transfuge malgré soi: l'Homme de qualité hésite entre Guillaume d'Orange et Jacques II, et fuit pour éviter ce dilemme: ce n'est pas grave. Montcal, obligé de passer en Angleterre à la suite d'un duel, se retrouve dans les troupes anglaises; heureusement, il est protestant (IV: 253); il n'en combattra pas moins contre son pays en Irlande, sous les ordres du maréchal de Schomberg. Celui-ci ayant servi successivement la Prusse, la Suède, la France, le Portugal et l'Angleterre, Montcal ne peut choisir meilleur modèle; le voici excusé, d'autant plus que Schomberg, lui, a agi par intérêt et «égarements du coeur» (CP 301-302). A un tel niveau de gloire, on ne saurait évidemment parler de trahison: il s'agit de choix politique. Pourtant, dans sa trilogie de 1740, Prévost s'intéresse à ces étonnantes équivoques: l'ambassadeur Ferriol mène à Constantinople un jeu de despote oriental; le Commandeur de Malte vit en libertin et passe auprès de l'Ordre pour un renégat; dans les Mémoires de Malte, on voit aussi apparaître des figures plus inquiétantes: Junius a abandonné l'Ordre pour fonder à Maina un petit royaume soumis au Grand Seigneur (IV: 171); il est vrai qu'il refuse de se marier et d'embrasser le mahométisme. Antonio, lui, est un vrai traître, passé au service du roi du Maroc et renégat de surcroît, pénible dans son abjection (IV: 197); 
d'une certaine façon, cela rassure. Les détestables transfuges se signalent en effet par leur cupidité, leur absence de scrupule et leur mépris de la religion; ils incarnent le mal absolu. Les demi-transfuges, les indécis, les libertins, les égarés ne font qu'illustrer les faiblesses de la nature humaine. Il en résulte que le cynisme des renégats provoque l'horreur, alors que l'hypocrisie des opportunistes (Montcal, le Commandeur) est finalement justifiée.

\section{Frontières}

Ce sont là d'indécises limites, qui autorisent tous les doubles jeux; mais ce qui intéresse le plus Prévost, c'est précisément le passage, ou la transgression d'une frontière. Les héros de ses romans sont des exilés, des fugitifs, des transfuges; ses héroïnes sont presque toutes immigrées; ce sont des Turques ou des Grecques (Sélima, Théophé), des Anglaises ou des Irlandaises (Fanny, Rose), des Espagnoles (Dona Cortona), des Italiennes (Helena), des Hongroises (Mlle Tekeli); et encore faudrait-il mentionner les Françaises transplantées à l'étranger, comme Manon. La qualité d'exilé ou d'émigré, peut-on croire, ajoute quelque chose à la personnalité, ou en montre un aspect inconnu. De l'autre côté de la frontière, un être va se révéler, dans son originalité ou dans son malheur. D'où l'importance du passage. Le lieu de la frontière est souvent mis en scène: c'est la frontière du Nord de la France, entre la Flandre française et la Flandre espagnole dans les Mémoires d'un homme de qualité; c'est encore le trajet entre Londres et Feversham, ou le passage obligé de Calais, qui prête à tant de variations chez Prévost, ${ }^{8}$ Constantinople est un port où arrivent des inconnus, ou d'où l'on s'échappe en un instant, un lieu de marché ou de rencontres; au cours des voyages seront notés les ports, les escales, les îles. Dans ces ports, des agents officieux, des maîtres de langue, des espions: l'un des personnages de Prévost est un consul des Echelles du Levant, riche d'expériences étonnantes.'

$\mathrm{D}^{\prime}$ un côté à l'autre de la frontière se produit un changement intérieur du héros, une métamorphose. L'Homme de qualité hésitait entre les hanovriens et les jacobites; le voici transformé en défenseur de la chrétienté et en route pour l'inconnu. Une fois passé en Turquie, il connaîtra une seconde métamorphose: à la découverte de l'amour s'ajoutera celle d'un autre mode de vie. On pourrait le croire tout à coup converti à une autre civilisation, dont il découvre avec étonnement les aspects humains et raffinés. Personne, à vrai dire, ne peut résister à cette connaissance quasiment sensualiste: à Rouen, le jeune Cleveland devient un être différent; à Paris, il se montrera incapable de résister à l'appel du luxe. Les frères et la soeur du doyen de Killerine, à peine arrivés à Paris, sont saisis par un climat d'abondance et de plaisir: 
Ce spectacle fut nouveau pour nous, qui n'avions vu jusqu'alors que de la pauvreté et de la misère dans les villes désertes d'Irlande. Je remarquai d'une manière sensible l'effet que ce changement produisit sur mes frères et sur ma soeur. (III: 26)

On observerait la même transformation chez des Français établis en Angleterre: l'Homme de qualité et son disciple Rosemont sont gagnés par la vitalité londonienne et ne quittent l'Angleterre qu'avec regret. Prévost lui-même avouait qu'il ne pensait plus de la même façon dès qu'il avait passé la Manche. ${ }^{10}$

Inversement, il arrive plus d'une fois que l'immigrant apporte à son nouveau pays une culture nouvelle. L'Homme de qualité, prisonnier des Turcs, s'efforce de gagner leur sympathie en leur faisant découvrir l'équitation, le jardinage, et la belle littérature, grâce à une traduction de Télémaque qu'il répand en manuscrit (I: 71). Le catéchisme n'est pas oublié, mais on constate qu'il prête désormais à une comparaison avec le Coran (I: 74-75). Prêt à comprendre une autre nation et à s'en faire comprendre, le héros ne refuse pas d'en acquérir la langue ou de l'apprendre à celle qu'il aime, situation d'échange que l'on n'avait guère connue dans le roman classique. ${ }^{11}$ Il arrive que par l'effet de cette emprise sentimentale et culturelle la métamorphose mène au drame. Théophé, soustraite à son milieu d'origine, devient autre et accède à une vie morale qu'elle n'avait jamais connue; mais la liberté idéale qu'elle conquiert ne lui servira jamais à rien, puisqu'elle ne s'accompagne pas d'un changement réel de condition. On pourrait observer chez son amant, l'ambassadeur Ferriol, une transformation inverse: captivé par la Turquie, dont il connaissait la langue au départ, il s'est comme fondu dans sa nouvelle nation:

... et la seule singularité de voir un Français aussi turc, si l'on me permet cette expression, que les habitants naturels du pays, m'attira dès les premiers jours des caresses et des distinctions dont on ne s'est jamais relâché. (IV: 11)

Cette fascination n'est pas sans danger: devenu aussi turc que les Turcs, Ferriol semble en prendre peu à peu les coutumes et l'état d'esprit, dans ce qu'il a de plus étranger aux moeurs françaises. Il sera jaloux et despotique comme un Turc, entraîné à enfermer Théophé dans une sorte de sérail, à se comporter, à Marseille ou à Paris, comme un tyran. Le récit nous fait assister ainsi à une double métamorphose par emprunts culturels, mais sans incidence sur la condition réelle: Théophé est devenue française, selon le code le plus idéaliste, tandis que Ferriol est devenu turc, selon le code le plus archaïque. L'une reste l'esclave, et l'autre le maître. Cette situation limite appartient à la fiction tragique. Dans ses 
deux romans historiques, Prévost décrit une sorte d'assimilation en profondeur, mais qui procède de la politique plutôt que des changements de mentalité, qu'il n'aborde pas. Marguerite d'Anjou oublie qu'elle est française et ne songe plus qu'à «faire renaître dans ses états l'abondance avec la tranquillité» (V: 129). Déjà Guillaume le Conquérant, une fois établie sa domination, avait songé avant tout au «soulagement de la nation anglaise» (V: 319). Mais il faudra plusieurs siècles avant que les Normands et les Saxons fassent un seul peuple.

Prévost s'est donné souvent comme un médiateur, et plus précisément dans Le Pour et Contre, revue dans laquelle il essaie, pendant huit ans, de faire comprendre la culture anglaise au public français; mais plus encore que la culture, il tente d'approcher un mode de perception spécifiquement anglais:

Il me paroît fort étrange que dans un siècle où chaque Nation se flate, \& peut-être avec raison, d'être plus avancée que jamais dans les belles connoissances, on se ressemble si peu sur mille choses qui en dépendent, telles que le goût \& les sentimens, les moeurs, \&c. (PC V: 6)

D'une nation à l'autre, malgré les ressemblances culturelles' et les connaissances partagées, il reste de l'intraduisible: on passe la frontière, on pense autrement, on saisit la réalité de son propre pays de loin et sous un autre angle. C'est cette nouvelle optique que Prévost essaie de faire passer dans sa revue. Mais cette expérience ne peut s'exprimer le plus souvent que par la fiction.

\section{Retours}

Le voyage ne sert pas à découvrir de nouveaux paysages, mais à changer d'être. C'est pourquoi dans les voyages de Prévost on ne peut rien fonder; on ne peut que fuir, changer, vieillir. Dans les voyages bien réels de Robert Lade ou dans l'Histoire des voyages, on conquiert, on civilise, on spécule, on gagne un certain bonheur. Dans les romans, jamais. On part à contrecoeur, parce qu'on est ruiné, chassé, condamné, exclu; et l'on part sans désir d'aventure, comme le dit l'Homme de qualité:

... je n'avais point de goût pour cette multitude de courses et d'aventures bonnes et mauvaises qui sont inévitables à une personne qui s'expatrie. (I: 51)

Il part en songeant à l'heure de sa mort, puisqu' «il en faudra revenir là». Son immense périple va se dérouler comme en spirale; il parcourt le monde tout en revenant périodiquement à l'asile qu'il a choisi pour sa fin. Une grande boucle lui fait parcourir la Hollande, l'Angleterre, l'Alle- 
magne, la Turquie, l'Italie, pour le ramener au pays de ses ancêtres. Une seconde boucle le conduira d'Espagne en Portugal, puis en Hollande et en Angleterre, avant le retour dans son abbaye. Curieusement, le récit ne nous dit rien de ce centre caché. Par déduction, on comprend que la famille de l'Homme de qualité résidait en Artois; que le corps de Julie, sa soeur, y a été ramené; que lui-même reviendra dans le château familial, avant de se retirer dans une abbaye proche (I: 102). Le centre caché de son périple serait donc le lieu où est enterré l'inoubliable Julie, sa soeur morte à seize ans. ${ }^{12}$ Les voyages de Cleveland ont un caractère analogue: il a quitté la grotte de Rumney Hole, en Devonshire, pour parcourir l'Amérique, l'Atlantique et la France; mais il ne peut se fixer ni chez les Abaquis, ni à Cuba, ni à Saint-Cloud; et quand sa fille Cécile meurt, il décide de regagner l'Angleterre, pour la transporter en Devonshire (II: 637). ${ }^{13}$ Dans Manon Lescaut, le double projet de ce voyage en spirale s'explicite: Des Grieux, faute d'avoir pu mourir sur le corps de Manon, revient dans sa famille, en Artois. ${ }^{14} \mathrm{D}^{\prime}$ autres voyages évoqués dans les romans finissent plus mal encore: le narrateur des Mémoires d'un honnête homme est comme emmuré dans une forteresse d'Innsbruck, parmi des gardiens dont il ne comprend pas la langue; l'abbé Brenner est mort à la Bastille après avoir rédigé le long récit qui nous est transmis. $C^{\prime}$ est dire que d'une façon ou d'une autre ces relations nous mènent à la conclusion de l'Homme de qualité: «il en faudra revenir là».

Prévost n'a jamais accordé beaucoup d'importance à l'idée de patrie, qui ne convient guère, selon lui, aux gens de qualité:

... il ne put résister à l'amour de la patrie, qui a des charmes pour tout le monde, suivant le langage du poète, mais des charmes invincibles pour les âmes du commun. (VI: 302) ${ }^{15}$

Pour lui, il est plus important d'être fidèle à sa religion ou à son honneur, ou à l'amour, qu'au pays dont on est le sujet. Il attache cependant de l'importance au retour au pays natal, et le fait de s'expatrier garde pour lui une connotation malheureuse, les plus malheureux étant ceux qui ne reverront jamais leur pays. ${ }^{16}$

Ce serait naturellement simplifier les choses que de réduire les itinéraires prévostiens à ce trajet régressif ou fatal. Ce qui fait la variété de ces fuites, de ces passages de frontières, de ces périples orientés, c'est aussi l'espoir de trouver dans l'exil un asile. Aussi trouve-t-on dans ces longues errances des haltes, des séjours heureux, des utopies. On observe pourtant que chacun de ces épisodes se referme sur lui-même: les protestants de La Rochelle, dans Cleveland, ont cru trouver à SainteHélène un séjour de paix, de justice, d'affection familiale; mais ce séjour est comme frappé de malédiction par la stérilité qui les atteint, ${ }^{17}$ comme 
si le développement de l'humanité exigeait le mouvement, le conflit, les passions. L'utopie des Nopandes est plus troublante: cette petite nation douce et civilisée semble être parvenue à arrêter le temps; on y vénère en portrait un seigneur espagnol du siècle précédent; on y exerce une justice un peu archaïque mais immuable; on y vit simplement, en pratiquant la morale naturelle; le fils du prince, amoureux de Cécile, qui n'a que deux ans, ne souhaite que de la tenir sur ses genoux. Il faudra quitter ce petit peuple parfaitement bon, et l'on abandonnera le petit prince endormi sur les rivages de la Floride, en sachant qu' on le condamne au malheur (II: 555-556). Chaque utopie entrevue annonce aussitôt la déception: on peut toujours espérer retrouver le bonheur de l'enfance, une mère bienveillante, une famille pacifique, ou l'image d'un frère qui tient sa petite soeur sur ses genoux, ce ne sont que des images trompeuses, et ce qu'on croyait être l'avenir appartient au passé révolu. On espérait retarder le cours de l'histoire, on ne l'arrête pas, et son cours est toujours dramatique.

Le voyage commençait par une exclusion et une fuite, avec un regard en arrière sur le paradis perdu; il s'est poursuivi à regret, en s'attardant sur des escales entrevues; il s'achève sur un retour au havre familial, mais vu sous la forme d'une crypte. Alors que le voyage réel impliquait une découverte, une conquête, et des bénéfices tangibles, rien ne semble subsister du voyage de la vie. Et pourtant, de cette odyssée malheureuse, on ne peut oublier les acquis profonds. De tous, le plus précieux est certainement la connaissance des autres, ces échanges délicats par lesquels, lentement, l'autre cesse d'être un étranger. Persuadé que chaque nation possède son style de vie, son accès particulier au réel, son génie propre, Prévost s'est efforcé de faire passer dans la fiction ces modes d'existence. Il était d'instinct traducteur, médiateur, ethnographe: le Pour et Contre et l'Histoire des voyages le montrent amplement. Par ses romans, il prétend aller plus avant dans le «monde moral», un monde dans lequel apparaissent aussi les différences entre les peuples, et qui n'est plus celui des moralistes classiques. D'où ces fugitifs, ces transfuges, et ces immigrés, d'où encore cette fascination pour l'étranger, pour l'expérience de la transgression ou de la médiation. Sans ces passages de frontières, sans ces métamorphoses successives, il n'y aurait pas de progression intérieure. Le prisonnier qui nous livre à la fin son expérience des voyages est immobilisé, crispé sur un passé dramatique, mais sans la découverte des autres et l'évocation de ce qu'ils étaient, il n'y aurait pas de sens à son existence, et pas de récit.

\section{JEAN SGARD}

Université Stendhal (Grenoble) 


\section{Notes}

1 Sur le nom de «Prévost d'Exiles,» voir notre article «Antoine Prévost d'Exiles».

2 Toutes nos références renvoient aux Oeuvres de Prévost (tome et page entre parenthèses dans le texte). Les sigles de renvoi aux titres de romans, comme dans notre Prévost romancier, sont les suivants: MHQ (Mémoires d'un homme de qualité), ML (Manon Lescaut), C (Cleveland), DK (Le Doyen de Killerine), GM (Histoire d'une Grecque moderne), CP (Campagnes philosophiques), JC (Histoire de la jeunesse du Commandeur), MHH (Mémoires d'un honnête homme), RL (Voyages de Robert Lade), $M M$ (Le Monde moral), PC (Le Pour et Contre). Nous rassemblons sous la notion de «trilogie de 1740» GM, CP et $J C$, et comme romans historiques l'Histoire de Guillaume le Conquérant et l'Histoire de Marguerite d'Anjou.

3 La dispersion à la suite d'un traité apparaît au début de $M H Q$ (I: 13). Le remariage du père apparaît dans $M H Q$ (I: 17, 49), dans $M H H$ (VI: 212, 226), $M M$ (VI: 290). La fuite en secret des amants est présente dans $M H Q$ (I: 16, 320), dans $M L$, dans DK (III: 315), dans JC (154). Quant aux duels malheureux, ils sont présents dans toutes les oeuvres.

4 Sur la disgrâce de Clarendon, voir Prévost romancier 134. Cleveland, lui, redoute surtout les jésuites et leur allié Gelin, qui le blesse d'ailleurs grièvement (II: 352).

5 C. E. Engel a naguère attiré l'attention sur l'importance du thème du Refuge dans l'oeuvre de Prévost (73-125).

6 Voir en particulier le cas de Mme de $\mathrm{R}^{* * *}$, épouse d'un jacobite anglais dans $M H Q$ (I: 233), Mme de Gien dans CP (IV: 258), ou l'histoire du trésor de Jacques II dans DK (III: 372-375).

7 Prévost distingue nettement les apostats hésitants des renégats, qui font acte de soumission à une nouvelle religion. Voir en particulier MHQ I: 66, 76. L'appartenance à une religion reste pour lui la première marque d'identité sociale; plus encore, le masque de l'hypocrisie dénature l'individu.

8 Voir notre article «A l'enseigne du Lion d'or» (295-314).

9 Le Consul du Levant a ramené de Syrie deux femmes, une Grecque et une Géorgienne, ce qui pose des problèmes d'insertion assez délicats; $1^{\prime}$ Homme de qualité provoque le mariage de l'une et l'entrée au couvent de l'autre (I: 103-114).

10 Le Pour et Contre (PC), tome III, p.160-161.

11 Voir notre article «L'amour interprète».

12 Nous avons abordé cette question dans Labyrinthes de la mémoire (106-107).

13 «Sur nos terres de Devonshire», dit le texte, donc sur les terres héritées par Fanny de son père Axminster.

14 On sait que Des Grieux, au lieu de débarquer au Havre pour aller ensuite à Paris, où l'attend en principe le Séminaire, se rend à Calais pour rejoindre un parent, en Artois ou en Picardie.

15 Le poète mentionné est Voltaire, dans Tancrède; voir la note de R. Favre dans les Oeuvres de Prévost, VIII: 486.

16 S'expatrier, selon le Manuel lexique de Prévost, c'est «sortir de son Païs natal, y renoncer, pour aller vivre dans un autre païs», éd. 1763, I:385. 


\section{Jean Sgard}

17 La colonie des Rochelois a été fondée par des protestants; elle ressemble plutôt à une petite société de quakers, sage, équilibrée, apparemment durable. L'île dépérit toutefois par manque d'hommes (II: 107), ce qui sera la source du drame.

\section{Textes cités}

Engel, C[laire] E[liane]. Figures et aventures du XVIIIe siècle: voyages et découvertes de l'abbé Prévost. Paris: éd. «Je sers», 1939.

Prévost, Antoine, abbé. Oeuvres de Prévost. Réd. Jean Sgard. 8 vol. Grenoble: Presses universitaires de Grenoble, 1978-1986.

.Manuel lexique. Paris: éd. Didot, 1763.

. Le Pour et Contre. 20 vol. Paris: éd. Didot, 1733-1740.

Sgard, Jean. L'abbé Prévost: labyrinthes de la mémoire. Presses universitaires de France, 1986.

Prévost romancier. Paris: Corti, 1968, 1989.

ed. Vingt études sur Prévost d'Exiles. Grenoble: ELLUG, 1995.

"A l'enseigne du Lion d'or». Dans Vingt études. 295-314.

. «Antoine Prévost d'Exiles». Vingt études. 11-23.

. «L'amour interprète». Il Genio delle lingue. Roma: Treccani, 1989. 